\title{
Abnormal radioiodine uptake on post-therapy whole body scan and sodium/iodine symporter expression in a dermoid cyst of the ovary: report of a case and review of the literature
}

Alfredo Campennì', Salvatore Giovinazzo ${ }^{2}$, Giovanni Tuccari ${ }^{3}$, Simone Fogliani ${ }^{4}$, Rosaria M. Ruggeri ${ }^{2}$, Sergio Baldari ${ }^{1}$

\section{SUMMARY}

In patients affected by differentiated thyroid cancer, the whole-body scan (WBS) with 131-radioiodine, especially when performed after a therapeutic activity of ${ }^{131}$, represents a sensitive procedure for detecting thyroid remnant and/or metastatic disease. Nevertheless, a wide spectrum of potentially pitfalls has been reported. Herein we describe a 63-year-old woman affected by follicular thyroid cancer, who was accidentally found to have an abdominal mass at post-dose WBS (pWBS). pWBS showed abnormal radioiodine uptake in the upper mediastinum, consistent with lymph-node metastases, and a slight radioiodine uptake in an abdominal focal area. Computed tomography revealed an inhomogeneous mass in the pelvis, previously unrecognized. The lesion, surgically removed, was found to be a typical dermoid cyst of the ovary, without any evidence of thyroid tissue. By immunohistochemistry, a moderate expression of the sodium-iodine symporter (NIS) was demonstrated in the epithelial cells, suggesting a NIS-dependent uptake of radioiodine by the cyst. Arch Endocrinol Metab. 2015;59(4):351-4

\author{
1 Dipartimento di Scienze Biomediche e \\ delle Immagini Morfologiche e Funzionali, \\ Unità di Medicina Nucleare, Università \\ degli Studi di Messina, Messina, Italia \\ 2 Dipartimento di Medicina \\ Clinica e Sperimentale, Unità di \\ Endocrinologia, Università degli \\ Studi di Messina, Messina, Italia \\ ${ }^{3}$ Dipartimento di Patologia \\ Umana, Università degli Studi \\ di Messina, Messina, Italia \\ ${ }^{4}$ Unità di Scienze Radiologiche. \\ Ospedale di Milazzo, Messina, Italia \\ Correspondence to: \\ Alfredo Campennì \\ Dipartimento di Scienze Biomediche \\ e delle Immagini Morfologiche \\ e Funzionali, U.O.C. di \\ Medicina Nucleare, \\ A.O.U. Policlinico \\ 'G. Martino' di Messina, \\ v. Consolare Valeria, 1, \\ 98125 - Messina, Italy \\ acampenni@unime.it \\ Received on Apr/23/2014 \\ Accepted on Aug/21/2014
}

DOI: $10.1590 / 2359-3997000000087$

\section{INTRODUCTION}

$\mathrm{D}$ ifferentiated thyroid cancer (DTC), accounting for about $90 \%$ of all thyroid cancers, represents the most common endocrine malignancy and its incidence has increased in worldwide population (1). The initial treatment for DTC patients is total or near-total thyroidectomy, with or without lymph nodes dissection $(2,3)$. Radioiodine aid the management of DTC patients who have undergone surgical treatment, and its role in the diagnosis and treatment of such patients is well established $(2,3)$. Whole-body scan (WBS) with radioiodine, especially when performed after a therapeutic activity of ${ }^{131} \mathrm{I}$, represents an important diagnostic tool, in particular in intermediate or high risk DTC patients (2-4). Indeed, post-therapy WBS (pWBS) is a highly sensitive procedure for detecting residual normal thyroid tissue as well as metastases in local lymph nodes or distant sites (2-4). Nevertheless, false-positive scans, showing the presence of ${ }^{131} \mathrm{I}$ uptake in the absence of residual thyroid tissue or metastases can occur in different situations. Beside healthy tissues (i.e. salivary glands, breast, liver, and gastrointestinal tract), unexpected radioiodine uptake have been seen in benign diseases, such as cysts and inflammation, as well as in benign and malignant non-thyroidal tumors, which could be mistaken for metastases (5). Several potential pitfalls in the interpretation of WBS have been reported in the literature (5). Some of them are relatively common (6), while others are very uncommon in our experience (7-9). Herein we describe a case of ${ }^{131} \mathrm{I}$ accumulation in an ovarian dermoid cyst accidentally discovered at pWBS in a DTC patient.

\section{CASE REPORT}

A 63-year-old woman underwent near-total thyroidectomy for a large multi-nodular goiter. Histopatho- 
logical reports revealed a follicular thyroid carcinoma with minimal vascular invasion (pT3, N0, Mx). After conventional L-T4 withdrawal, the patient underwent radioiodine thyroid uptake (RTU) measurement, 24 hours after oral administration of ${ }^{131} \mathrm{I}$ tracer activity (1.8 $\mathrm{MBq}$ ), followed by radioiodine therapy (RIT) with ablative activity (3700 MBq). A post-dose ${ }^{131}$ I whole body scan (pWBS) was performed five days after RIT, using dual-headed gamma camera equipped with high-energy low-resolution parallel-hole collimator (HELRPAR). pWBS showed abnormal and intense radioiodine uptake in the upper mediastinum, consistent with lymph-node metastases (Figure 1, panels A and B, and a slight radioiodine uptake in an abdominal focal area located on the bladder activity (Figure 1, panels A and B, white arrow). At the time of RIT, serum TSH and Tg were $39.5 \mathrm{UI} / \mathrm{mL}$ and $546 \mathrm{ng} / \mathrm{mL}$ respectively. In absence of $\mathrm{TgAb}, \mathrm{Tg}$ levels were consistent with the presence of metastases. Computed Tomography (CT), performed before and after medium contrast administration, revealed a bilobed, inhomogeneous mass in the median region of the pelvis, extending to the upper wall of the bladder (Figure 1, panels C and D, white arrow). Our patient was unaware of this lesion and asymptomatic. A few weeks later, patient underwent surgical exploration with complete excision of the mass. Pathological examination was conclusive for a typical dermoid cyst of the ovary. No normal or tumoral thyroid tissue could be found in the context of the cystic lesion. A moderate expression of the sodium-iodine symporter (NIS) was demonstrated in the epithelial cells lining the cystic cavity, by immunohistochemistry (anti-NIS antibody SLC6A3, MyBioSource.Com, San Diego, CA, USA; working dilution, 1:25) (Figure 2). Immunostaining for hTg was negative, thus confirming the absence of thyroid tissue in the context of the dermoid cyst, as per gross pathology evaluation.

A few months later, the patient underwent a second RIT by administration of ${ }^{131}$ I therapeutic activity ( 5550 $\mathrm{MBq}$ ) in hypothyroid state (after five weeks of L-T4 withdrawal). pWBS confirmed lymph-node metastases in the mediastinum (Figure 3, black arrow), but did not reveal any radioiodine uptake in abdomen, confirming that radioiodine uptake was only caused by the dermoid cyst. At the time of the second RIT, serum $\mathrm{TSH}$ and Tg were $112 \mathrm{UI} / \mathrm{mL}$ and $242 \mathrm{ng} / \mathrm{mL}$, respectively. TgAb were negative.

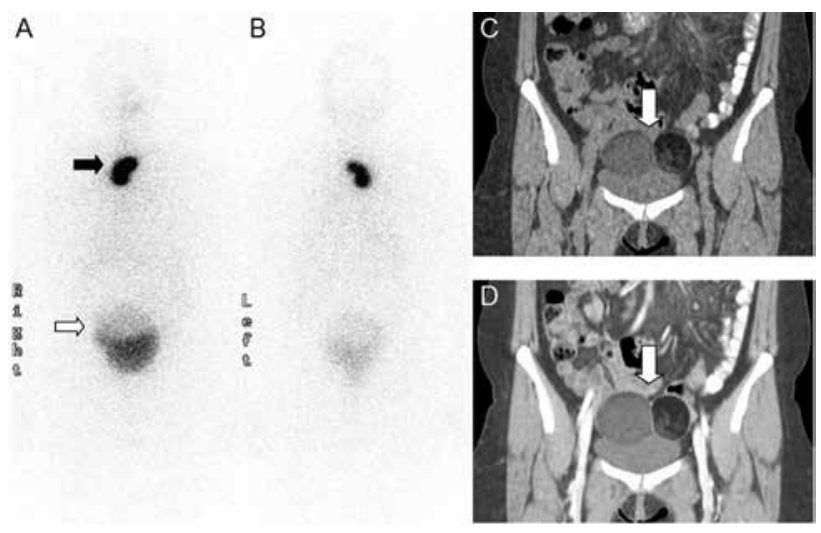

Figure 1. Panels $\mathbf{A}$ (anterior view) and $\mathbf{B}$ (posterior view). Post-therapy ${ }^{131}$ | whole body scan, showing intense and abnormal radioiodine uptake in a focal area located in the upper mediastinum (black arrow) and a focal area of slight radioiodine uptake in the inferior abdominal region, immediately above the bladder activity (white arrow). Panels $\mathbf{C}$ and $\mathbf{D}$. Tomography Computed scan obtained without (C) and with contrast agent (D), revealing a bilobed, inhomogeneous mass in the median region of the pelvis, extending on the upper wall of the bladder (white arrows).

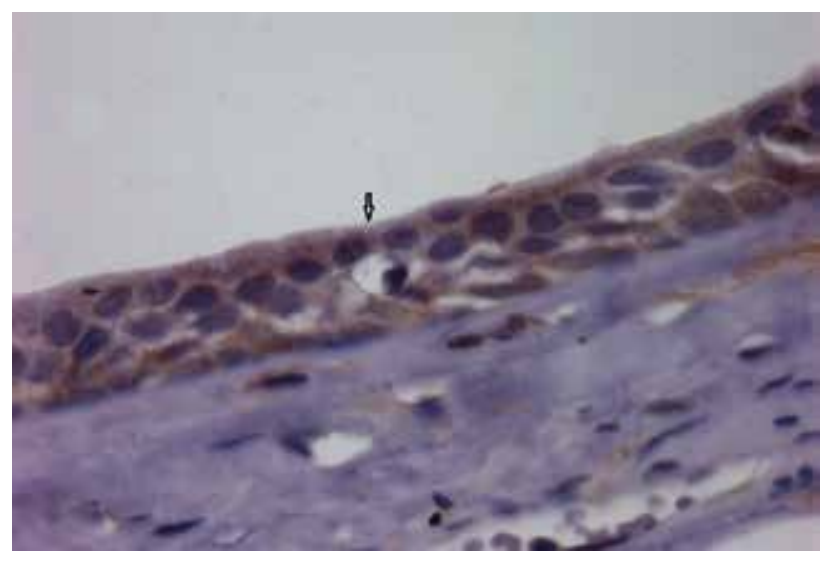

Figure 2. Immunohistochemical detection of the sodium-iodine symporter (NIS) in the dermoid cyst. A diffuse, slight to moderate immunostaining was encountered in the cubic epithelium lining the cystic lesion (original magnification, 300X; Mayer's haemalum counterstain).

\section{DISCUSSION}

In patients affected by DTC, the post-therapy ${ }^{131} \mathrm{I}$ WBS represents a sensitive procedure for detecting thyroid remnant and/or metastatic disease (2-4). Nevertheless, a wide spectrum of potentially misleading readings has been reported following its use (5).

Multiple different false-positive localizations of ${ }^{131} \mathrm{I}$ has been described in the absence of residual thyroid tissue or metastases from DTC (5-9). The underlying mechanisms of such unusual localizations are not always fully understood. In many cases, the iodine uptake may be due to the expression of the sodium/ iodine symporter (NIS) in ectopic thyroid tissue as 
A

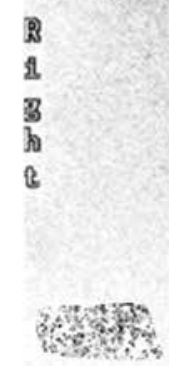

Figure 3. Panels $\mathbf{A}$ (anterior view) and $\mathbf{B}$ (posterior view). ${ }^{131}$ w whole body scan confirmed abnormal radioiodine uptake in the upper mediastinum (black arrow), but did not confirm abdominal radioiodine uptake.

well as in non-thyroidal normal or pathological tissues $(5,10-12)$. In some instances, foci of ${ }^{131}$ I activity are not related to the presence of NIS, but due to retention of radioiodine in physiologic body fluids accumulated in ducts or cavities $(13,14)$ as well as contamination by physiologic secretions (5). In addition, it has also reported radioiodine trapping in various inflamed tissues and/or cysts because of their increased vascularity and capillary permeability (15-17). Finally, in some cases, the mechanism for ${ }^{131} \mathrm{I}$ trapping remains unknown. Unless recognized as a false positive, ${ }^{131}$ I uptake may result in diagnostic error and lead to unnecessary ${ }^{131} \mathrm{I}$ therapy or surgical procedures.

Here we report an unexpected and potentially misleading ${ }^{131} \mathrm{I}$ uptake in the abdomen, revealed at the pWBS, in a DTC patient. The CT scan showed the presence of a bilobed, inhomogeneous mass in the median region of the pelvis. The lesion, previously unrecognized and asymptomatic, was surgically removed, and it was found to be a typical dermoid cyst of the ovary, without any evidence of thyroid tissue nor tumour cells.

Dermoid cysts, also known as teratomas, are germ cells tumours, histologically composed of mature tissues that develop from ectoderm, mesoderm and/ or endoderm. Typical dermoid cysts are epithelial-lined cavities with skin appendages, including hair, hair follicles, sebaceous material, deriving from ectodermal tissue. They may also contain mesodermal tissue (bone, teeth...), and tissue of endodermal origin, including well differentiated thyroid tissue (18). Dermoid cysts primarily occur in the gonads, mostly in female patients. However, dermoids are not unique to a single anatomic location, but may occur anywhere in the body. Moreover, they are often clinically silent, so that the patient is unaware of this malformation, as in our case.

Ovarian radioiodine uptake at radioiodine WBS, although so uncommon, has been previously reported (19). However, it usually occurs in germ line tumours which contain well-differentiated thyroid tissue and just because they contain it. As shown in the literature (18), up to $20 \%$ of ovarian teratomas contain thyroid tissue, and $5 \%$ of them are struma ovarii, defined as the tumour being more than half thyroid tissue $(18,19)$. Struma ovarii represent an uncommon but well known false-positive localization of ${ }^{131} \mathrm{I}$ (20-22), because it is mainly or exclusively composed of well-differentiated and functioning thyroid tissue, able to trap iodine as well as to produce thyroid hormones and thyroglobylin (18-22). Up to date, there are only four reports in the literature of radioiodine uptake by ovarian dermoid cysts, not be classified as struma ovarii (23-26).

Rudoni and cols. first reported on a 44-year old woman who underwent total thyroidectomy for a papillary cancer. The pWBS demonstrated a focal uptake in the pelvis, that was found to be due to a typical dermoid cyst of the ovary (23). After this first report, three other cases were described (24-26). All were childbearing-age female patients, who have been previously undergone total thyroidectomy for DTC, two papillary and one follicular cancer. In all patients, as in our, the ovarian lesion was clinically silent and previously unrecognized, and it was incidentally discovered on WBS in the follow-up of the thyroid cancer. The WBS showed an abnormal pelvic uptake, associated with an elevated $\mathrm{Tg}$ value just in one patient (26). Thus, the patients underwent further imaging evaluation (CT or magnetic resonance imaging), showing an ovarian lesion, followed by the surgical removal of the mass. The histological examination was always conclusive for a benign dermoid cyst (not struma ovarii). In all except one (23) of these cases, the lesion contained a variable amount of benign, well-differentiated thyroid tissue, thus justi- 
fying the radioiodine uptake. Prior of us, just Rudoni and cols. reported ${ }^{131}$ I uptake in a ovarian dermoid cyst, that was totally devoid of thyroid tissue at histological diagnosis, as in our case, but the Authors did not provide any possible explanation of such unusual finding (23). We first demonstrated the expression of NIS in the epithelial cells of the cystic lesion, suggesting that radioiodine uptake was NIS-dependent, and not related to the increase of vascularity or capillary permeability.

In conclusion, we describe a very uncommon case of false positive ovarian uptake of radioiodine in a WBS for DTC, due to a typical dermoid cyst expressing the NIS on its epithelial cells. Due to the non-negligible frequency of this malformation, dermoids should be taken into account in DTC patients as a potential pitfall at ${ }^{131} \mathrm{I}-\mathrm{WBS}$.

Statement of authorship: each author gave a substantial contribute to the paper and approved the final version to be published.

Funding statement/competing interest: this work was not supported by any grant.

Disclosure: no potential conflict of interest relevant to this article was reported.

\section{REFERENCES}

1. Pellegriti G, Frasca F, Regalbuto C, Squatrito S, Vigneri R. Worldwide increasing incidence of thyroid cancer: update on epidemiology and risk factors. J Cancer Epidemiol. 2013;2013:965212.

2. Pacini F, Schlumberger $M$, Dralle $H$, Elisei $R$, Smit JW, Wiersinga W; European Thyroid Cancer Taskforce. European consensus for the management of patients with differentiated thyroid carcinoma of the follicular epithelium. Eur J Endocrinol. 2006;154(6):787-803.

3. American Thyroid Association (ATA) Guidelines Taskforce on Thyroid Nodules and Differentiated Thyroid Cancer, Cooper DS, Doherty GM, Haugen BR, Kloos RT, Lee SL, Mandel SJ, et al. Revised American Thyroid Association management guidelines for patients with thyroid nodules and differentiated thyroid cancer. Thyroid. 2009;19(11):1167-214.

4. Pacini F. Follow-up of differentiated thyroid cancer. Eur J Nucl Med Mol Imaging. 2002;29 Suppl 2:S492-6.

5. Oh JR, Ahn BC. False-positive uptake on radioiodine whole-body scintigraphy: physiologic and pathologic variants unrelated to thyroid cancer. Am J Nucl Med Mol Imaging. 2012;2(3):362-85.

6. Kayano D, Michigishi T, Ichiyanagi K, Inaki A, Kinuya S. I-131 uptake in a thymic cyst. Clin Nucl Med. 2010;35(6):438-9.

7. Campenni A, Minutoli F, Pecorella GR, Baldari S. Unusual radioiodine uptake in a patient with thyroid cancer and cranial metallic clips. Nuklearmedizin. 2002;41(6):N89-N90.

8. Baldari S, Sturniolo G, Violi MA, Moleti M, Campennì A, Calbo E, et al. Meningocele due to closed spina bifida mimicking a metastasis of papillary thyroid carcinoma on whole body radioactive iodine scan. Thyroid. 2011;21(12):1401-2.
9. Campennì A, Ruggeri RM, Giovinazzo S, Sindoni A, Santoro D, Baldari S. Radioiodine uptake in a renal cyst mimicking a metastasis in a patient affected by differentiated thyroid cancer: case report and review of the literature. Ann Nucl Med. 2014;28(5):472-6.

10. Shapiro B, Rufini V, Jarwan A, Geatti O, Kearfott KJ, Fig LM, et al. Artifacts, anatomical and physiological variants, and unrelated diseases that might cause false-positive whole-body $131-I$ scans in patients with thyroid cancer. Semin Nucl Med. 2000;30(2):115-32.

11. Spitzweg C, Joba W, Eisenmenger W, Heufelder AE. Analysis of human sodium iodide symporter gene expression in extrathyroidal tissues and cloning of its complementary deoxyribonucleic acids from salivary gland, mammary gland, and gastric mucosa. J Clin Endocrinol Metab. 1998;83(5):1746-51.

12. Tazebay UH, Wapnir IL, Levy O, Dohan O, Zuckier LS, Zhao QH, et al. The mammary gland iodide transporter is expressed during lactation and in breast cancer. Nat Med. 2000;6(8):871-8.

13. Wolff $H$, Breda DJ, Da Silva N, Hartmann AA. False-positive $\mathrm{I}-131$ deposition in a parotid gland duct ectasia. Clin Nucl Med. 1998;23(4):257-9.

14. Maslack MM, Wilson CA. lodine131 accumulation in a pericardial effusion (letter). J Nucl Med. 1987;28(1):133.

15. Triggiani V, Moschetta M, Giagulli VA, Licchelli B, Guastamacchia E. Diffuse 1311 lung uptake in bronchiectasis: a potential pitfall in the follow-up of differentiated thyroid carcinoma. Thyroid. 2012;22(12):1287-90.

16. Brucker-Davis F, Reynolds JC, Skarulis MC, Fraker DL, Alexander HR, Weintraub BD, et al. False-positive iodine-131 whole-body scans due to cholecystitis and sebaceous cyst. J Nucl Med. 1996;37(10):1690-3.

17. Bural GG, Peel RL, Mountz JM. Benign epithelial cyst mimicking thyroid cancer metastasis: a false-positive finding on post-therapy I-131 scan. Clin Nucl Med. 2012;37(1):88-90.

18. Wolff EF, Hughes M, Merino MJ, Reynolds JC, Davis JL, Cochran $\mathrm{CS}$, et al. Expression of benign and malignant thyroid tissue in ovarian teratomas and the importance of multimodal management as illustrated by a BRAF-positive follicular variant of papillary thyroid cancer. Thyroid. 2010;20(9):981-7.

19. Anastasilakis $A D$, Ruggeri RM, Polyzos $S A$, Makras $P$, Molyva $D$, Campennì $A$, et al. Coexistence of Graves' disease, papillary thyroid carcinoma and unilateral benign struma ovarii: case report and review of the literature. Metabolism. 2013;62(10):1350-6.

20. Zwas ST, Heyman Z, Lieberman LM. 131I ovarian uptake in a whole-body scan for thyroid carcinoma. Semin Nucl Med. 1989;19(4):340-2.

21. Salvatori M, Rufini V, Daidone MS, Danza FM, Valentini AL, Negro $\mathrm{F}$, et al. [Occasional detection of "struma ovarii" in a patient with thyroid carcinoma]. Radiol Med. 1991;81(5):744-7.

22. Jammah AA, Driedger A, Rachinsky I. Incidental finding of ovarian teratoma on post-therapy scan for papillary thyroid cancer and impact of SPECT/CT imaging. Arq Bras Endocrinol Metabol. 2011;55(7):490-3.

23. Rudoni S, Toubeau M, Vaillant G, Arnould L, Vergès B, Brun JM, et al. [Dermoid cyst of the ovary. False positive diagnosis in iodine 131 scintigraphy of differentiated thyroid cancers]. Presse Med. 1998;27(27):1379-81.

24. Basu S, Mahajan A. Ovarian dermoid cyst serendipitously detected by pelvic radioiodine-(131)l uptake and by diffusion weighted MRI in a post-thyroidectomy case of papillary thyroid carcinoma. Hell J Nucl Med. 2013;16(1):62-3.

25. van Wijk JP, Broekhuizen-de Gast HS, Smits AJ, Schipper ME, Zelissen PM. Scintigraphic detection of benign ovarian teratoma after total thyroidectomy and radioactive iodine for differentiated thyroid cancer. J Clin Endocrinol Metab. 2012;97(4):1094-5.

26. Gauthé M, Kelly A, Dejax C, Cachin F, Bourdel N. Incidental scintigraphic finding of ovarian teratoma containing normal thyroid tissue on post-radioactive iodine therapy for papillary thyroid cancer. Clin Nucl Med. 2013;38(6):467-8. 